\title{
Intrathecal Gadolinium-Enhanced MR Cisternography: A Comprehensive Review
}

\author{
O. Algin and B. Turkbey
}

\section{ABSTRACT}

SUMMARY: CE-MRC has been in use for the past 15 years and was reported to be a useful method in the evaluation of CSF disorders and hydrocephalus. The use of CE-MRC in conjunction with other MR imaging techniques has been shown to be effective in selected cases for the evaluation of several disorders of cerebrospinal system. CE-MRC has certain advantages over other cisternographic studies with fewer side effects if performed properly. Although intrathecal Gd administration is not widely accepted yet, several recent studies have reported the safety of small-dose intrathecal gadolinium injection. In this review, we describe CE-MRC and review recent applications in several clinical conditions.

ABBREVIATIONS: $\mathrm{AC}=$ arachnoid cyst; $\mathrm{AS}=$ aqueductal stenosis; $\mathrm{CE}=$ contrast-material enhanced; $\mathrm{CISS}=$ constructive interference in steady state; $\mathrm{CTC}=\mathrm{CT}$ cisternography; CTM = CT myelography; ETV = endoscopic third ventriculostomy; $\mathrm{Gd}=$ gadolinium; Gd-DTPA = gadopentetate dimeglumine; IHS = intracranial hypotension syndrome; ICP = intracranial pressure; MRC = MR cisternography; $\mathrm{NCE}=$ noncontrast-material enhanced; $\mathrm{MRM}=\mathrm{MR}$ myelography; $\mathrm{NPH}=$ normal pressure hydrocephalus; $\mathrm{PC}=$ phase-contrast; $\mathrm{RC}=$ radionuclide cisternography; $\mathrm{STV}=$ spontaneous third ventriculostomy

C isternography is defined as imaging of the cerebrospinal system and associated structures after intrathecal administration of contrast material into the subarachnoid space. ${ }^{1,2}$ Cisternographic studies have been in use for at least 50 years for the evaluation of abnormalities of the intracranial CSF-filled spaces, cranium base, and vertebral column; however, it is called "myelography" if it is dedicated to spinal canal investigation only. ${ }^{1,2}$ In this review, different imaging techniques performed after intrathecal contrast agent administration will be called "cisternography."

The main limitations of RC are lack of cross-sectional images and lower spatial resolution. CT has overcome these limitations; however, both CTC and RC involve radiation exposure, which is a more important factor to consider because cisternographic studies often require consecutive or multiple acquisitions. ${ }^{2-6}$

NCE-MRC derived from heavily 3D T2-weighted sequences (such as fast imaging with steady state acquisition, CISS, or sampling perfection with application optimized contrast using different flip angle evolutions) has been developed to overcome the

Received August 24, 2011; accepted after revision September 1.

From the Department of Radiology (O.A.), Atatürk Training and Research Hospital, Bilkent, Ankara, Turkey; and National Cancer Institute (B.T.), National Institutes of Health, Bethesda, Maryland.

Please address correspondence to Oktay Algin, MD, Department of Radiology, Atatürk Training and Research Hospital, Bilkent, Ankara, Turkey; e-mail: droktayalgin@gmail.com

--m Indicates open access to non-subscribers at www.ajnr.org

http://dx.doi.org/10.3174/ajnr.A2899 above-mentioned limitations of CTC and RC. ${ }^{3-5}$ This technique can be helpful in investigating CSF leakage and the fluid dynamics of the ventricular system. ${ }^{7-10}$ High contrast-to-noise ratio, multiplanar evaluation of volumetric data, and thin-section image acquisition are the main advantages of NCE-MRC. ${ }^{11}$ Moreover, it is noninvasive, and it does not require radiation exposure or intrathecal contrast material administration. ${ }^{4}$ Although NCEMRC has several advantages, it can only provide morphologic information different from that in other cisternographic studies. $^{9,10}$

CE-MRC can provide both physiologic and morphologic information, unlike NCE-MRC. ${ }^{6}$ CE-MRC is a less invasive technique compared with other cisternographic and ventriculographic (radionuclide or MR ventriculography) tests, and it enables use of all the advantages of MR imaging. ${ }^{9} \mathrm{CE}-\mathrm{MRC}$ can be easily performed with other MR imaging examinations (such as PC-MR imaging).

Intrathecal Gd injection is not approved by the FDA, though its safety has been reported in several pilot studies in Europe. ${ }^{2-4,6,9-14}$ Tolerance and dose limits of intrathecal Gd injection were established in animal model studies, and different $\mathrm{Gd}$ compounds (eg, gadodiamide, gadobenate dimeglumine, and Gd-DTPA) were compared in different experimental animal protocols. ${ }^{5,14-17}$ High-dose intraventricular Gd injection was reported to result in behavioral and neurologic disturbances (focal seizures, ataxia, latent tremor) with histopathologic changes (loss of oligodendroglia, astrocytic hypertrophy, and eosinophilia); on the other hand, it was concluded that these changes and distur- 

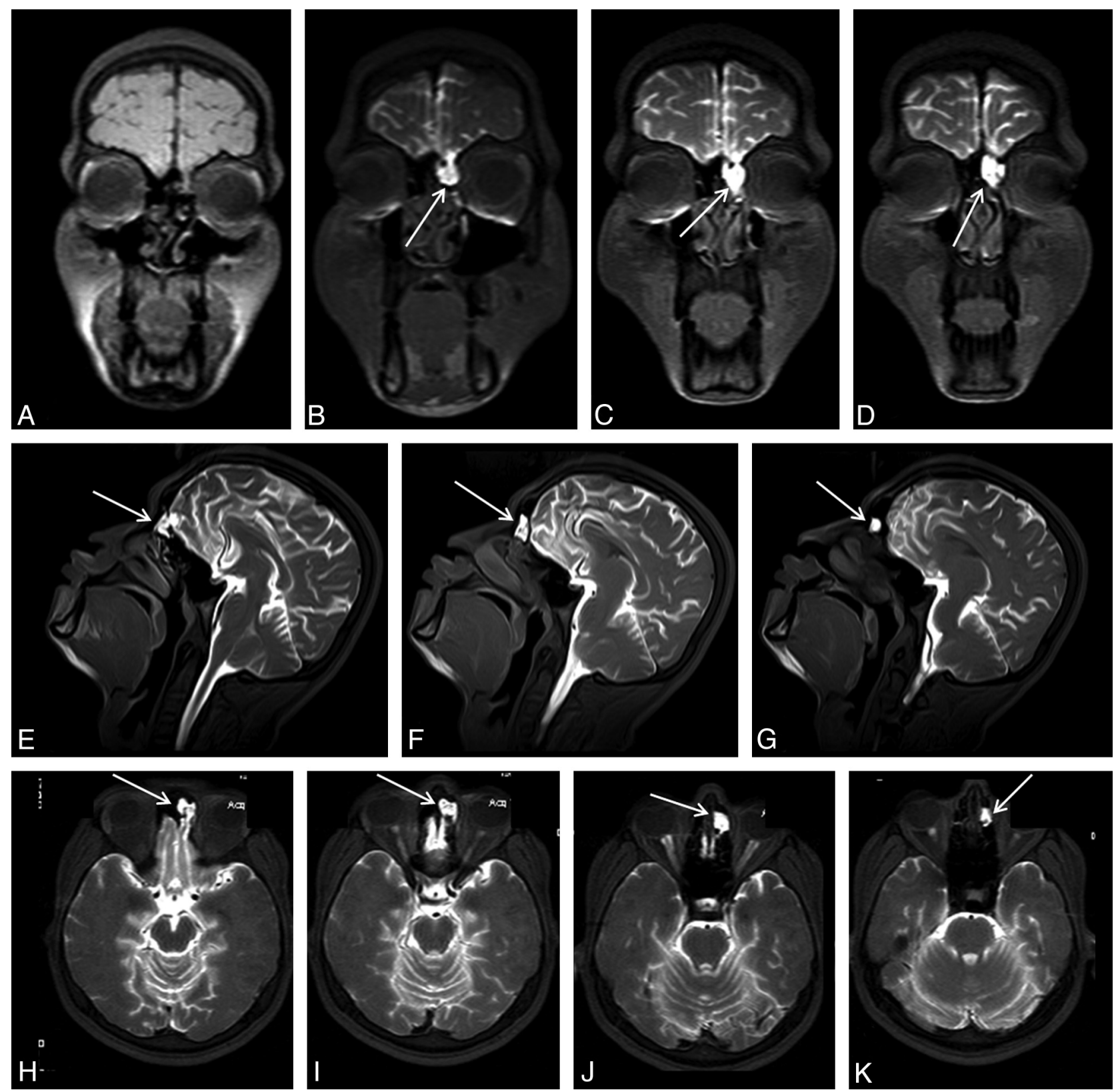

FIG 1. An 18-year-old man with posttraumatic recurrent meningitis. Coronal precontrast TIWI shows irregularity and heterogeneity of the left cribriform plate $(A)$. Early-phase postcontrast TIWI demonstrates passage of the contrast material from left cribriform plate through anterior ethmoid cells (arrows in B-K). Presence of the left cribriform plate defect was confirmed at surgery.

bance did not occur if the total injected dose was $<15 \mathrm{~mL}(3.3$ $\mathrm{mmol} / \mathrm{g}) .^{18-20}$

The first clinical human study for intrathecal Gd injection included 2 patients with meningeal carcinomatosis; this was followed by other trials, which reported the safety of intrathecal Gd. ${ }^{21}$ In these studies, no significant change was observed on physical and neurologic examinations, electroencephalography, and CSF findings of patients following CE-MRC with $<1 \mathrm{~mL}$ of intrathecal Gd injection. ${ }^{2,6,9,10,12-17,22-26}$ Gd-DTPA has been reported as the safest and most recommended intrathecal contrast agent in human studies. ${ }^{2,4,6,9,10}$ In our cohort of approximately 100 patients who had CE-MRC for at past 7 years in 2 different centers, we have not encountered a major complication. Headache was the most commonly encountered postprocedural minor complication in our cohort.

\section{TECHNIQUE OF CE-MRC}

Before intrathecal Gd administration for CE-MRC, precontrast 3-plane T1-weighted and 3D heavily T2-weighted (NCE-MRC) images are obtained. In patients with a leakage work-up, T1weighted images should be fat-suppressed; otherwise fat-suppression it is not mandatory. However, all precontrast and postcontrast images should be obtained with the same parameters. ${ }^{4}$ 


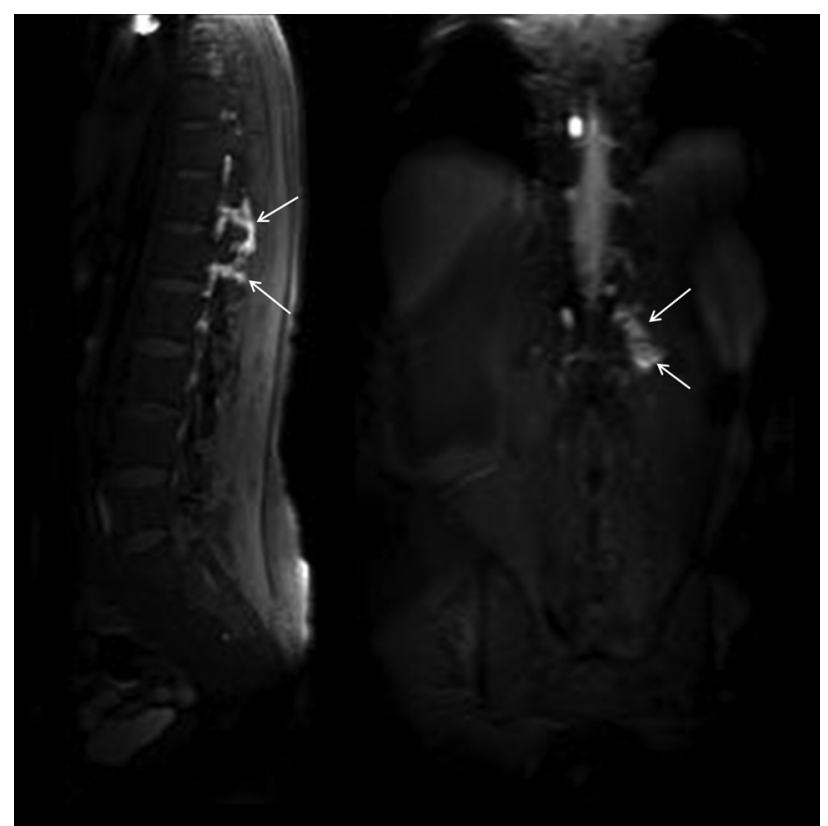

FIG 2. A 42-year-old man with spontaneous intracranial hypotension syndrome. Postcontrast early-phase sagittal (left) and coronal (right) TIWI shows leakage of contrast material into the paraspinal space at the thoracolumbar junction level (arrows). In this patient, epidural blood patch was planned.

In our clinic, CE-MRC studies are performed with 2D (such as TSE) or 3D (such as 3D-fast low angle shot or volumetric interpolated breath-hold sequence) T1-weighted sequences. Volumetric data obtained from single-plane 3D sequences with isotropic voxels by $3 \mathrm{~T}$ MR imaging machines allow thin-section and multiplanar evaluation, which significantly reduces the examination time. ${ }^{11}$

After acquisition of precontrast T1-weighted and T2-weighted images, $0.5 \mathrm{~mL}$ (for adults) of Gd-DTPA is diluted 2-fold with CSF and injected into the subarachnoid space through a 24- to 26-ga needle/1-mL syringe under proper sterile conditions. ${ }^{25,26}$ CE-MRC can be used for children, but the optimal dose of GdDTPA is unclear in the literature. ${ }^{13}$ The lower lumbar region (L4-L5) is usually preferred for intrathecal administration; whereas injection can be performed through the lateral portions of the $\mathrm{C} 1$ or C2 vertebral body. ${ }^{9,16}$ Also, the reservoir of a shunt system can be used for injection. ${ }^{13}$ In centers with limited experience, performing lumbar puncture under fluoroscopic guidance can reduce the incidence of complications and side effects.

Acquisition time of postcontrast T1weighted images depends on the underlying pathologies that are being investigated. We recommend early-phase (within the first 6 hours) imaging for all patients. Early-phase images are helpful to see whether the contrast agent was successfully administered into the subarachnoid space and to evaluate spinal sub- arachnoid space and global CSF circulation. Postcontrast 1- to 2-hour imaging will be sufficient if spinal canal pathologies are investigated. ${ }^{1-3,27}$ Late-phase imaging is not mandatory for spinal canal pathologies, but it is necessary for the evaluation of intracranial CSF circulation.

Following CE-MRC, ideally all patients should be clinically observed for 48 hours; however, the patient can be discharged with a 2-day bed rest requirement if no symptom is experienced during or after CE-MRC. In a few patients, headache can occur secondary to dural puncture, and this usually resolves with bed rest and analgesic medication. ${ }^{4,9,10}$

\section{CLINICAL APPLICATIONS OF CE-MRC IN NEUROIMAGING}

Evaluation of Cranial CSF Leakage (Rhinorrhea-Otorrhea) Thin-section CT images can usually be helpful in visualizing the bony defects, but can be limited in patients with multiple fractures or intermittent CSF leakage. ${ }^{4}$ Additionally, the bony defect can sometimes be congenital, and leakage can occur from another location. The ability of CT to demonstrate the leakage site depends on indirect findings such as fracture lines within the cranium base, bony defects, mucosal abnormalities, the presence of fluid within the paranasal sinus, pneumocephalus, and meningoencephalocele. ${ }^{28,29}$ Thin-section CT and multidetector row $\mathrm{CT}$ have sensitivity and specificity values of $84 \%-95 \%$ and $57 \%-100 \%$, respectively, for depiction of leakage sites. ${ }^{4,30}$

$\mathrm{RC}$ and CTC have a limited value for detection of the leakage site. ${ }^{28-30}$ Moreover, routine use of CT alone or in conjunction with CTC or RC increases the radiation exposure significantly. ${ }^{4,28,29}$ The sensitivity of RC for the diagnosis of a CSF fistula is approximately $40 \%$, whereas it varies between $40 \%$ and $81 \%$ for CTC. ${ }^{6,30}$ Both techniques are quite limited in detecting low-flow fistula or hairlike communications. ${ }^{6}$

Leakage is characterized by a hyperintense tract between the subarachnoid space and the sinonasal spaces on NCE-MRC. ${ }^{22}$ Additionally, secondary parenchymal changes and associated en
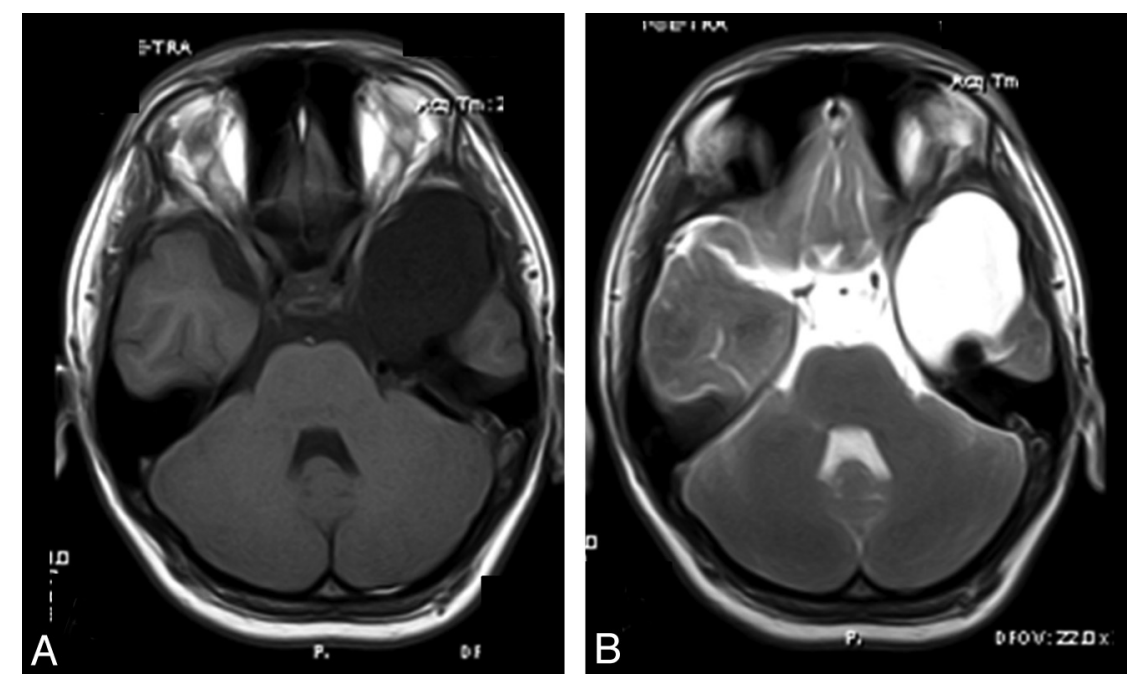

FIG 3. A 17-year-old boy with syncope history. Axial precontrast $(A)$ and early-phase postcontrast $(B)$ TIWI. Axial postcontrast TTWI shows opacification of the arachnoid cyst in the left temporal fossa $(B)$ consistent with a communicating type temporal arachnoid cyst. 

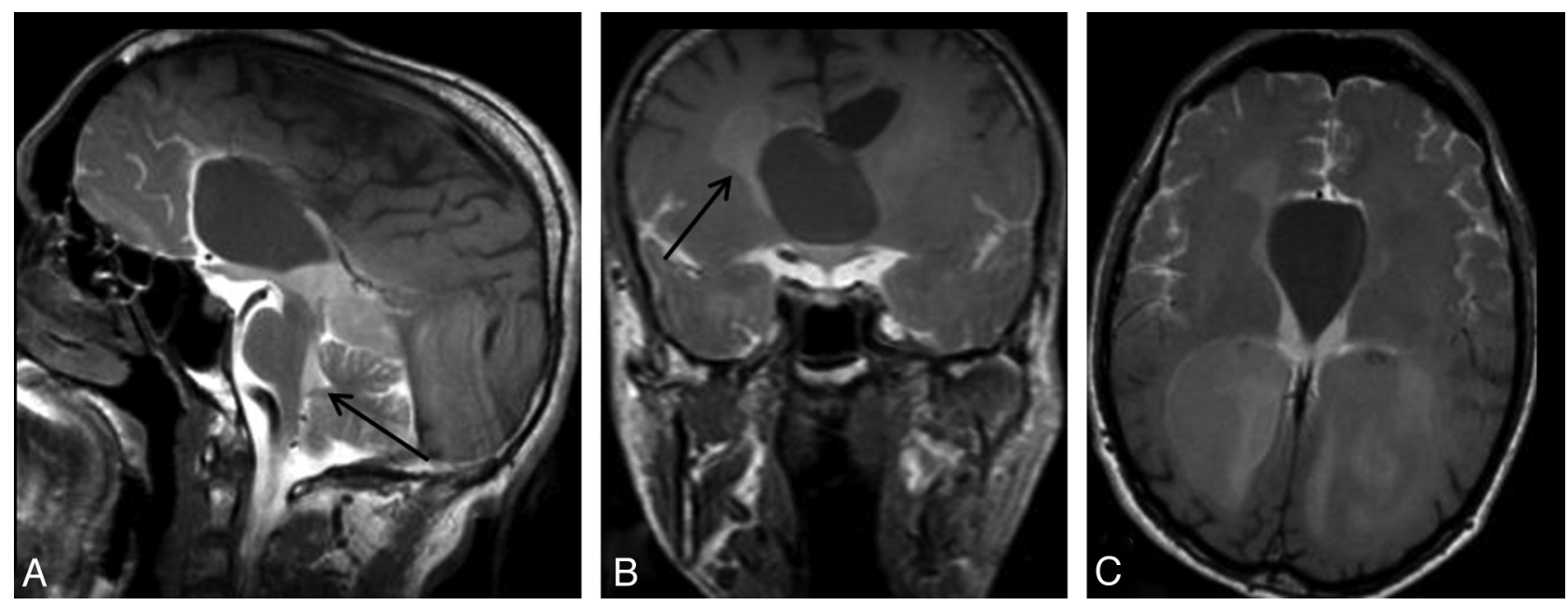

FIG 4. A 34-year-old man with headache. Sagittal early-phase postcontrast TIWI reveals an arachnoid cyst at the septum pellicidum (A). Early-phase postcontrast TIWI shows passage of the intrathecally administered contrast to the fourth ventricle (arrow) and basal cisterns, whereas there is no contrast in the arachnoid cyst $(A)$. Coronal $(B)$ and axial $(C)$ late-phase postcontrast images show passage of the contrast to the lateral ventricles (arrow in $B$ ), but not to the arachnoid cyst. Imaging findings are consistent with a non-communicating arachnoid cyst.

cephalocele suggestive of CSF leakage can be seen on NCE-MRC. ${ }^{4}$ However, these findings are not specific; sinusitis-mastoiditis, viscous secretions, and susceptibility artifacts secondary to the boneair interphase can result in increased false-positive diagnosis rates $(42 \%){ }^{6,29,30}$

On CE-MRC, visualization of hyperintense contrast leakage on fat-suppressed T1-weighted images is sufficient for the diagnosis of CSF leakage or fistula (Fig 1). ${ }^{27-29}$ Postcontrast images should be obtained in the second hour following intrathecal Gd administration. ${ }^{4}$ Additional postcontrast images should be obtained if no contrast is present within the mid or anterior cranial fossa. Valsalva maneuvers just before postcontrast image acquisition can be helpful in localizing the leakage site.

As a result, NCE-MRC can be chosen as a first-line imaging technique because there is no ionizing radiation exposure or contrast medium administration, and this technique is noninvasive and has a high soft-tissue contrast without artifacts from bony elements. A CT scan can be added to the algorithm if the patient has a positive trauma history. However, in more complicated situations such as in patients with positive results on a $\beta 2$-transferrin test but no visualized CSF leak or suspicious leak on NCEMRC images, CE-MRC should be performed. ${ }^{4}$

\section{IHS-Spinal CSF Leakage}

The main criterion for IHS diagnosis is demonstration of the CSF leakage, and it is important for treatment planning. ${ }^{31}$ Hence, epidural blood patch treatment can be more successful if applied to the leakage location directly. ${ }^{32}$

RC can directly demonstrate the leakage; however, lower softtissue resolution and lack of cross-sectional images are among its main disadvantages. ${ }^{2,6}$ Leakage of the radioisotope at the lumbar puncture site can result in false-positive results. ${ }^{27,31,33}$ CTC is more accurate in leakage localization, but patients are exposed to higher dose radiation $(>10 \mathrm{mSv}$ ) because thin sections have to be obtained from the skull base down to the sacrum. ${ }^{2}$ The high viscosity of CT contrast-media requires thicker needles (such as $20 \mathrm{ga}$ ) for lumbar puncture, which can decrease the accuracy and increase post- lumbar puncture - related complications such as iatrogenic dural defect, false-positive results, and headache. ${ }^{6,9,10,27}$

The NCE-MRM technique includes acquisition of thin-section heavily T2-weighted images similar to those in NCE-MRC. NCE-MRM is helpful in the detection of indirect findings such as epidural-paravertebral fluid and meningeal diverticula, which are strongly suggestive of a CSF leakage diagnosis. However, it may be inadequate to demonstrate a minimal but active CSF leakage. ${ }^{27}$ Moreover, a venous structure in the paravertebral region can mimic a CSF leakage, and proper interpretation of NCE-MRM requires experience.

CE-MRC (also called CE-MR myelography) is the most accurate test for visualization of a CSF fistula in patients with IHS. The reported sensitivity of CE-MRC in IHS is approximately $89 \%$, which is better than that of CTM (67\%), RC (55\%), NCE-MRM (86\%), and spinal MR imaging (50\%). 2,27,33 On postcontrast CEMRC images, the presence of leakage of the intrathecally administered Gd from the subarachnoid space to the epidural and/or paravertebral space is the most sensitive and direct finding of CSF leakage (Fig 2). ${ }^{27}$

Postcontrast CE-MRC images should be obtained in the early phase (within first 2 hours). ${ }^{27}$ Uniform contrast-material enhancement of the CSF can be achieved before imaging if the patient is asked to turn around a few times without standing or sitting up. Finally, imaging protocol of patients with IHS should include initial NCEMRM and T1-weighted (precontrast CE-MRC) series, and CE-MRC should be added to the protocol if a diagnosis cannot be reached or if indeterminate findings are present.

\section{Evaluation of AC Communication}

Determining the connection of ACs between CSF-containing spaces and their characterization is potentially important in surgical planning of symptomatic patients. ${ }^{14}$ Although conventional MR imaging sequences can provide sufficient information about cyst morphology, they are unable to demonstrate the connection of ACs with adjacent CSF spaces (Fig 4). ${ }^{34,35}$ CTC and RC can help to evaluate 

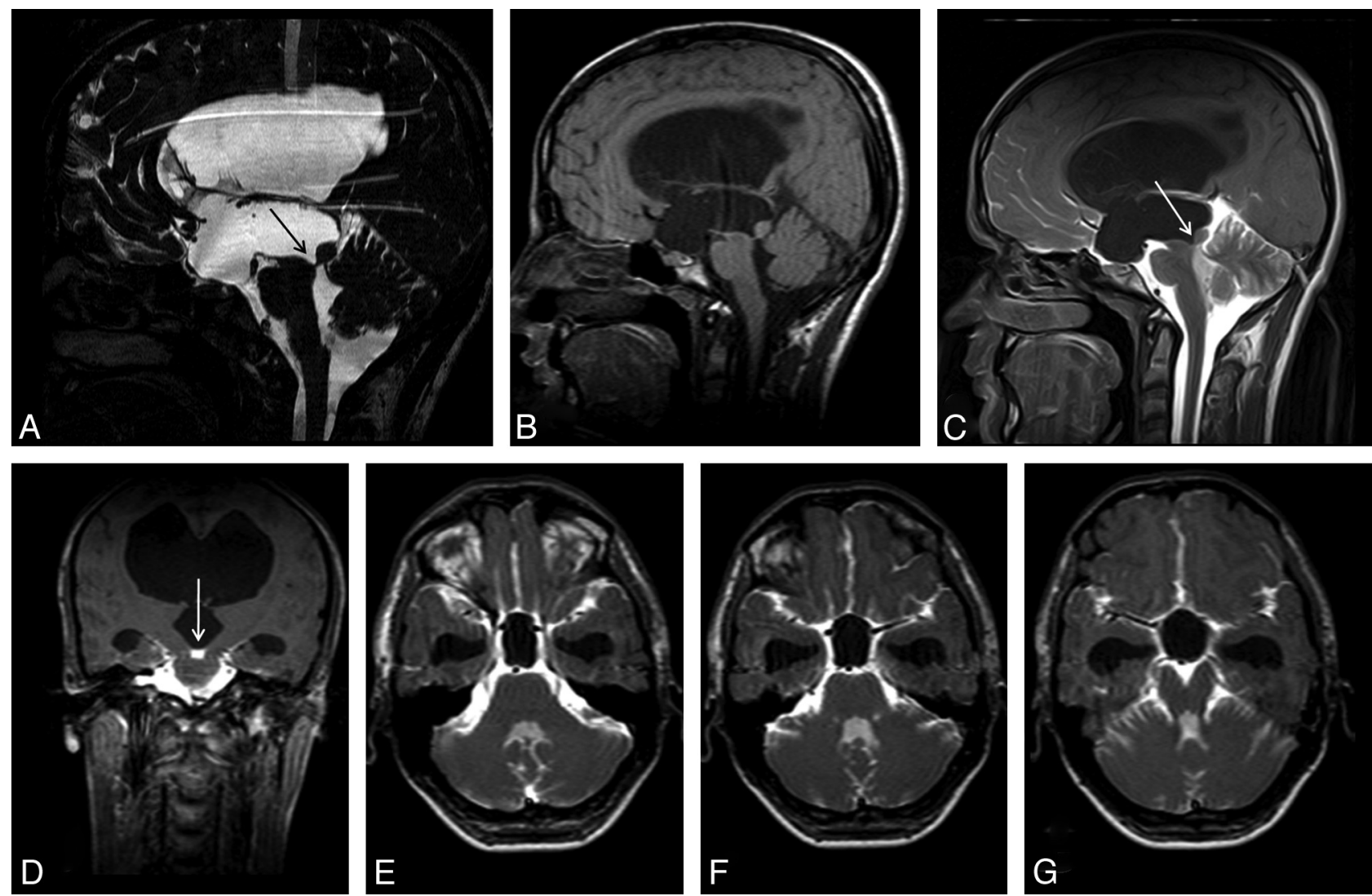

FIG 5. A 20-year-old man with headache. Sagittal 3D-CISS $(A)$ and precontrast TTWI (B) shows hydrocephalus. Sagittal 3D-CISS image demonstrates a linear hypointense band at the cerebral aqueduct (arrow in $A$ ). The inferior wall of the third ventricle cannot be depicted clearly on 3D-CISS image (A). Early-phase postcontrast sagittal and coronal TTWI shows passage of the intrathecally administered contrast to the fourth ventricle, but not to the third ventricle (arrows) (C, D). Imaging findings are consistent with aqueductal stenosis and hydrocephalus secondary to a web. A 12-hour post injection axial sequential TIWI showed no relation between the basal cisterns and the third ventricle (E-G). The intact third ventricular floor implies absence of a STV, and tells us ETV or shunt procedures are necessary.

connections between ACs and subarachnoid spaces; however, they have certain disadvantages as mentioned above. ${ }^{36}$

PC-MR imaging is a commonly used technique for the evaluation of AC and CSF connections. ${ }^{34,35}$ PC-MR imaging can demonstrate the presence of communications in ACs located at relatively stagnant or extraventricular regions such as the temporal fossa or convexity level; however, it is limited in locations with complex flow such as the intraventricular space or suprasellar cistern and can lead to false-positive results. ${ }^{36} 3 \mathrm{D}$ heavily $\mathrm{T} 2$-weighted sequences such as 3D-CISS can be helpful in determining cyst morphology and adjacent CSF-containing spaces; however, they do not provide sufficient information about communications. ${ }^{36}$

CTC and CE-MRC are accepted as criterion standard imaging techniques for the investigation of a communication between the $\mathrm{AC}$ and adjacent CSF spaces. ${ }^{34,35} \mathrm{CE}-\mathrm{MRC}$ is a more advantageous technique for this purpose compared with CTC. ${ }^{14,35} \mathrm{On}$ postcontrast CE-MRC images, the presence of contrast material within an AC is diagnostic (Fig 3). An AC is considered a noncommunicating type if there is no signal-intensity change between precontrast and postcontrast images (Fig 4). ${ }^{36}$

Contrast-material enhancement can occur at early or late phases of imaging in communicating ACs. ${ }^{14}$ Regardless of the size of the AC, the contrast-enhancement pattern can affect the surgical planning. ${ }^{36}$ Therefore, early- and late-phase postcontrast T1weighted images should be obtained in all patients with a differ- ential diagnosis of AC, and these images should be evaluated together.

\section{Aqueductal Stenosis}

Routine MR imaging sequences are usually limited in depicting the etiology of hydrocephalus and demonstrating the presence of AS (Fig 5). ${ }^{37}$ The basic criteria for diagnosis of AS in routine sequences are triventricular dilatation with a comparatively small fourth ventricle, narrowing of the aqueduct, and expansion of third ventricle borders and recess. ${ }^{9,38}$ However, most of these criteria are subjective; therefore, more effective techniques are needed. ${ }^{39}$ Additionally, MR imaging plays a critical role in treatment planning of patients with AS, specifically for selection of the appropriate surgical procedure (such as ventriculoperitoneal shunt, ETV, or endoscopic aqueductoplasty). 9,37,40

PC-MR imaging can provide physiologic information about CSF circulation but can lead to false results in the presence of complex flow or adjacent vascular pulsations. ${ }^{9}$ Additionally, PCMRI cannot provide morphologic data, and several technical factors (eg, inappropriate velocity-encoding value or wrong section position) can result in inadequate findings. ${ }^{9,10,38} 3 \mathrm{D}$-CISS and similar sequences have superior anatomic detail with higher spatial resolution; however, they cannot provide physiologic information about aqueductal flow. 9,40

There is no single accepted criterion standard MR imaging 

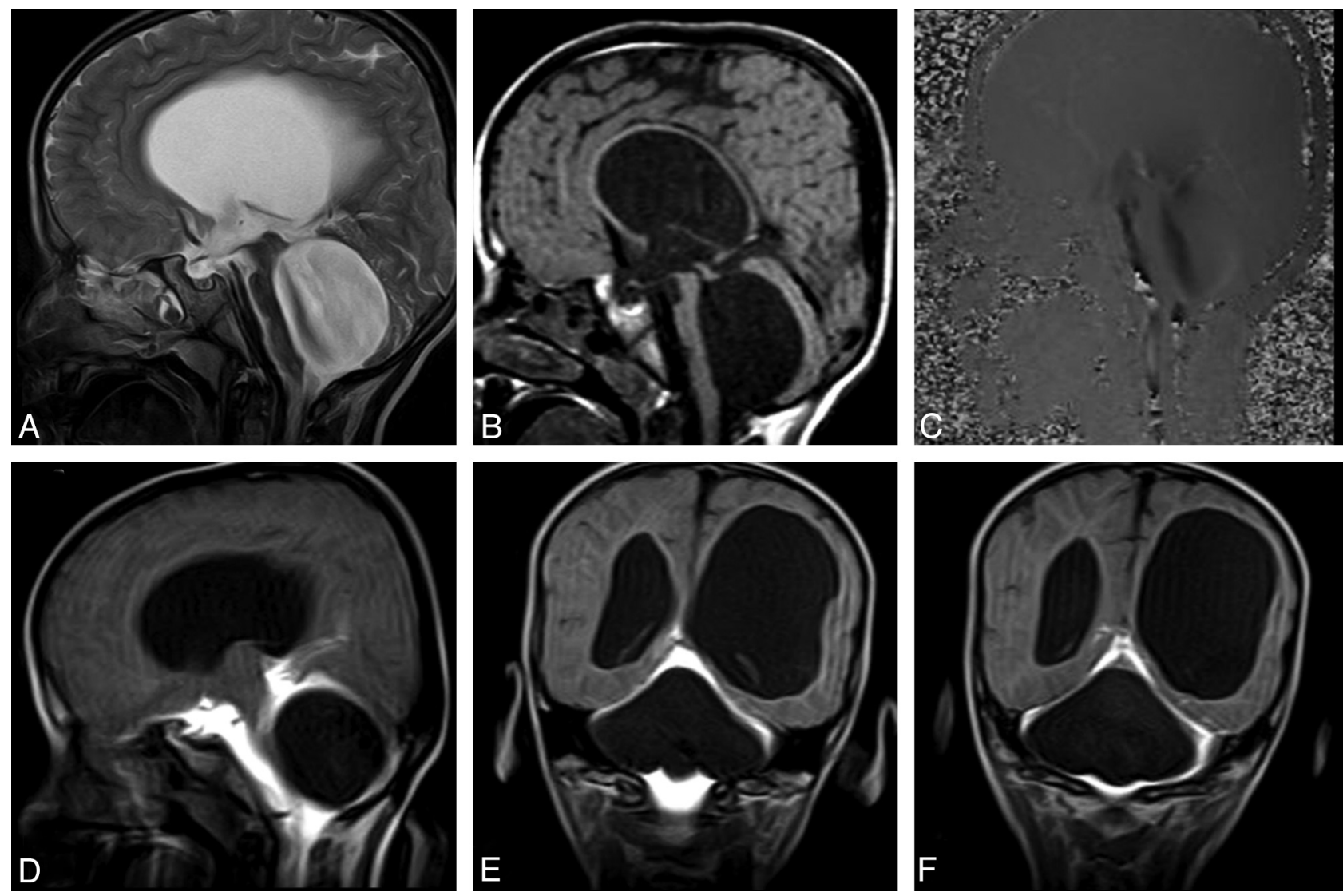

FIG 6. A 6-year-old boy with headache and syncope. Sagittal T2WI $(A)$ and precontrast TIWI $(B)$ show enlargement of the third and fourth ventricles with flattening of the corpus callosum. Sagittal PC-MR image demonstrates the black-coded flow at anterior fourth ventricle (C). Early-phase postcontrast TTWI clearly shows a non-communicating type cystic lesion leading to fourth ventricle enlargement (D-F).

protocol for the evaluation of patients with AS. ${ }^{9}$ Ventriculographic studies are accepted as criterion standard techniques for the diagnosis of AS; however, their invasive nature with severe complication risk precludes their routine clinical use. ${ }^{9} \mathrm{RC}$ and CTC, which are less invasive techniques, have been in use for diagnosis of AS, but their previously mentioned disadvantages are the main drawbacks; hence, CE-MRC is regarded as a better alternative for AS diagnosis work-up. CE-MRC is reported to prevent false results occurring in PC-MR imaging and 3D-CISS sequences. ${ }^{9} \mathrm{CE}-\mathrm{MRC}$ should be performed for AS diagnosis in cases in which PC-MR imaging shows partial obstruction or suspected flow and 3D-CISS displays a narrowed aqueduct. ${ }^{9}$

\section{Obstruction of the Fourth Ventricular Outlet}

Pathologic entities such as tumors, cysts, or infections leading to obstruction or stenosis of the fourth ventricular outlet can also result in hydrocephalus or fourth ventricle enlargement. ${ }^{34,37}$ The most common etiology for such obstructions is posterior fossa neoplasms in the pediatric age group, whereas it is membranousfibrous adhesions in adults. ${ }^{37,40} \mathrm{CE}-\mathrm{MRC}$ can be useful in the diagnosis and treatment planning if other noninvasive tests are limited (Fig 6).

\section{Evaluation of Spontaneous/Endoscopic Third Ventriculostomy}

STV is the rupture of the ventricular wall developing secondary to increased pressure of the ventricular system, usually as a result of obstructive hydrocephalus. ${ }^{41}$ As a result of STV, a direct communication between the ventricular system and subarachnoid space occurs. ${ }^{41,42}$ Forming a similar communication via endoscopic surgery is called "ETV." 39 The presence of an STV should be investigated before an ETV procedure because ETV is unnecessary in patients with STV. ${ }^{10}$ As a result, depiction of an STV is very important for treatment planning.

Ventriculographic studies are criterion standard techniques for evaluation of STV and ETV. ${ }^{10}$ However, their use is limited due to their invasiveness and severe complication risks, such as infection, increase in ICP (iatrogenic hypertension), brain injury, and intracranial hemorrhage; their use is limited in routine clinical practice. ${ }^{9,10,13,21}$

PC-MR imaging is the most commonly used technique in the investigation of the patency of ETV or the presence of STV. ${ }^{41,43}$ Detection of flow between the third ventricle and suprasellar cistern during both systole and diastole suggests the presence of an STV or ETV. ${ }^{44}$ The most important limitation of PC-MR imaging for ETV and STV evaluation is the false-positive results secondary to pulsation of the vascular structures adjacent to the third ventricle wall. $^{10,45}$

3D-CISS can provide useful morphologic information about third ventricle walls. ${ }^{40,43}$ However, in some patients, third ventricle wall evaluation can be limited if the walls are thin and close to adjacent structures as a result of increased ventricle pressure. ${ }^{10}$ 

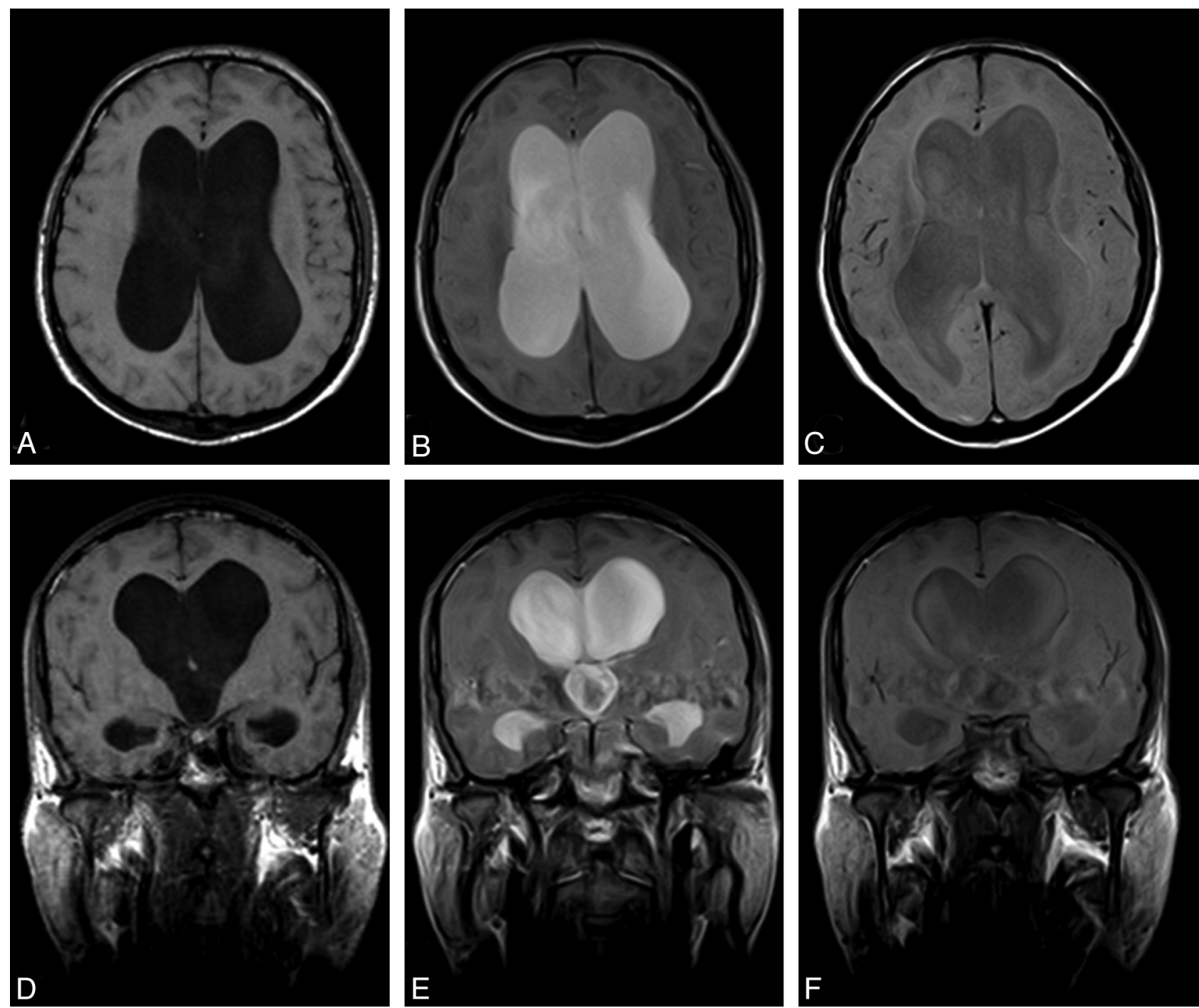

FIG 7. Positive CE-MRC examination of a 67 -year-old woman with definite NPH. Axial $(A)$ and coronal $(D)$ precontrast TIWI shows ventriculomegaly and effacement of the cisterns at convexity level. After intrathecal Gd-DTPA injection, the contrast material persisted in the lateral ventricles at $24(B, E)$ and $48(C, F)$ hours.

Such a complex anatomy can prevent optimal imaging of the third ventricle walls and the prepontine cistern (Fig 5). ${ }^{10,45}$

CE-MRC can be performed in patients who demonstrate suspected STV findings on PC-MR imaging and 3D-CISS sequences. ${ }^{10,45}$ CE-MRC may prevent false-positive results of PC-MR imaging and the 3D-CISS sequence. ${ }^{45}$ In patients with negative results on both PC-MR imaging and 3D-CISS sequences, CE-MRC may be unnecessary. ${ }^{10}$

\section{Normal Pressure Hydrocephalus}

$\mathrm{NPH}$ is a potentially treatable cause of dementia, and its accurate diagnosis is critical for treatment planning. ${ }^{46-49}$ Unfortunately, there is no single noninvasive gold standard test for the diagnosis of NPH. ${ }^{46-49}$ PC-MR imaging is a commonly used test in clinical practice. ${ }^{50}$ However, PC-MR imaging cannot always be conclusive for diagnosis and treatment planning. ${ }^{46}$

The persistence of intrathecally administered Gd within the lateral ventricles for 24 hours and even longer is a positive sign for the diagnosis of NPH (Fig 7).$^{48}$ Additionally, it was reported that patients with deficient ventricular clearance and with intraventricular Gd retention benefited more from CSF-diversion treatment. ${ }^{48} \mathrm{CE}-\mathrm{MRC}$ can be used in conjunction with routine MR imaging sequences and PC-MR imaging in diagnosis and treatment-response assessment of $\mathrm{NPH}^{48,49}$ Further largescale studies are needed to demonstrate the efficacy of CE$\mathrm{MRC}$ in NPH.

\section{CONCLUSIONS}

CE-MRC is a valuable MR imaging technique in the evaluation of hydrocephalus, CSF diversions, CSF leakages, central nervous system diseases (eg, tumors adjacent to CSF cavities), and abnormal CSF collections (eg, ACs). This technique can also allow evolution of CSF flow dynamics with the advantages of superior soft-tissue resolution, multiplanar imaging capability, and the absence of radiation exposure over CTC and RC. CE-MRC can be easily combined with other MR imaging techniques (such as PC-MR imaging and NCE-MRC). 


\section{ACKNOWLEDGMENT}

The authors thank Prof. Dr. Ergin Atalar, the director of National MR Research Center (UMRAM) for the opportunity he provided for 3T MR acquisitions. The authors gratefully acknowledge Dr. Ozgur Tosun, Prf. Dr. Halil Arslan, Prof. Dr. Mustafa Karaglanoglu, Prof. Dr. Bahattin Hakyemez, and Prof. Dr. Mufit Parlak for their contributions.

\section{REFERENCES}

1. Feld M, Clement J. Cisternography; technique, indications, results. Presse Med 1951;59:1396-400

2. Albayram S, Kilic F, Ozer H, et al. Gadolinium-enhanced MR cisternography to evaluate dural leaks in intracranial hypotension syndrome. AJNR Am J Neuroradiol 2008;29:116-21

3. Yoo HM, Kim SJ, Choi CG, et al. Detection of CSF leak in spinal CSF leak syndrome using MR myelography: correlation with radioisotope cisternography. AJNR Am J Neuroradiol 2008;29:649-54

4. Algin O, Hakyemez B, Gokalp G, et al. The contribution of 3D-CISS and contrast-enhanced MR cisternography in detecting cerebrospinal fluid leak in patients with rhinorrhoea. Br J Radiol 2010;83:22532. Epub 2009 Sep 1

5. Di Chiro G, Knop RH, Girton ME, et al. MR cisternography and myelography with Gd-DTPA in monkeys. Radiology 1985;157: 373-77

6. Selcuk H, Albayram S, Ozer $\mathrm{H}$, et al. Intrathecal gadolinium-enhanced MR cisternography in the evaluation of CSF leakage. AJNR Am J Neuroradiol 2010;31:71-75

7. Hatipoglu HG, Durakoglugil T, Ciliz D, et al. Comparison of FSE T2W and 3D FIESTA sequences in the evaluation of posterior fossa cranial nerves with MR cisternography. Diagn Interv Radiol 2007; 13:56-60

8. Kulkami M. Constructive interference in steady-state/FIESTA-C clinical applications in neuroimaging. J Med Imaging Radiat Oncol 2011;55:183-90

9. Algin O, Hakyemez B, Parlak M. Phase-contrast MRI and 3D-CISS versus contrast-enhanced MR cisternography on the evaluation of the aqueductal stenosis. Neuroradiology 2010;52:99-108

10. Algin O, Hakyemez B, Parlak M. Phase-contrast MRI and 3D-CISS versus contrast-enhanced MR cisternography for the detection of spontaneous third ventriculostomy. J Neuroradiol 2011;38:98-104

11. Watanabe Y, Makidono A, Nakamura M, et al. 3D MR cisternography to identify distal dural rings: comparison of 3D-CISS and 3DSPACE sequences. Magn Reson Med Sci 2011;10:29-32

12. Aydin K, Terzibasioglu E, Sencer S, et al. Localization of cerebrospinal fluid leaks by gadolinium-enhanced magnetic resonance cisternography: a 5-year single-center experience. Neurosurgery 2008;62:584-89

13. Muñoz A, Hinojosa J, Esparza J. Cisternography and ventriculography gadopentate dimeglumine-enhanced MR imaging in pediatric patients: preliminary report. AJNR Am J Neuroradiol 2007;28: 889-94

14. Tali ET, Ercan N, Kaymaz M, et al. Intrathecal gadolinium (gadopentetate dimeglumine)-enhanced MR cisternography used to determine potential communication between the cerebrospinal fluid pathways and intracranial arachnoid cysts. Neuroradiol 2004;46: 744-54

15. Krämer N, Berlis A, Klisch J, et al. Intrathecal gadolinium-enhanced MR-cisternography: depiction of the subarachnoidal space and evaluation of gadobenat-dimeglumin-(Gd-BOPTA, "Multihance") toxicity in an animal model and a clinical case. Acad Radiol 2002;9:S447-51

16. Tali ET, Ercan N, Krumina G, et al. Intrathecal gadolinium (gadopentetate dimeglumine) enhanced magnetic resonance myelography and cisternography: results of a multicenter study. Invest Radiol 2002;37:152-59

17. Park KW, Im SB, Kim BT, et al. Neurotoxic manifestations of an overdose intrathecal injection of gadopentetate dimeglumine. $J \mathrm{Ko}$ rean Med Sci 2010;25:505-08

18. Ray DE, Holton JE, Nolan CC, et al. Neurotoxic potential of gadodiamide after injection into the lateral cerebral ventricle of rats. AJNR Am J Neuroradiol 1998;19:1455-62

19. Ray DE, Cavanagh JB, Nolan CC, et al. Neurotoxic effects of gadopentetate dimeglumine: behavioral disturbance and morphology after intracerebroventricular injection in rats. AJNR Am J Neuroradiol 1996;17:365-73

20. Jinkins JR, Williams RF, Xiong L. Evaluation of gadopentetate dimeglumine magnetic resonance cisternography in an animal model: preliminary report. Invest Radiol 1999;34:156-59

21. Siebner HR, Gräfin von Einsidel H, Conrad B, et al. Magnetic resonance ventriculography with gadolinium DTPA: report of two cases. Neuroradiol 1997;39:418-22

22. Goel G, Ravishankar S, Jayakumar PN, et al. Intrathecal gadoliniumenhanced magnetic resonance cisternography in cerebrospinal fluid rhinorrhea: road ahead? J Neurotrauma 2007;24:1570-75

23. Arlt S, Cepek L, Rustenbeck HH, et al. Gadolinium encephalopathy due to accidental intrathecal administration of gadopentetate dimeglumine. J Neurol 2007;254:810-12

24. Li L, Gao FQ, Zhang B, et al. Overdosage of intrathecal gadolinium and neurological response. Clin Radiol 2008;63:1063-68

25. Jinkins JR, Rudwan M, Krumina G, et al. Intrathecal gadoliniumenhanced MR cisternography in the evaluation of clinically suspected cerebrospinal fluid rhinorrhea in humans: early experience. Radiology 2002;222:555-59

26. Zeng Q, Xiong L, Jinkins JR, et al. Intrathecal gadolinium-enhanced MR myelography and cisternography: a pilot study in human patients. AJR Am J Roentgenol 1999;173:1109-15

27. Algin O, Taskapilioglu O, Zan E, et al. Detection of CSF leaks with magnetic resonance imaging in intracranial hypotension syndrome. J Neuroradiol 2011;38:175-77

28. Stone JA, Castillo M, Neelon B, et al. Evaluation of CSF leaks: highresolution CT compared with contrast-enhanced CT and radionuclide cisternography. AJNR Am J Neuroradiol 1999;20:706-12

29. Wenzel R, Leppien A. Gadolinium-myelocisternography for cerebrospinal fluid rhinorrhoea. Neuroradiology 2000;42:874-80

30. Gulcek SY, Erdogan A, Toprak U, et al. Evaluation of rhinorrhea by computed tomography cisternography [in Turkish]. Tani Girisim Radyol 2003;9:327-32

31. Schick U, Musahl C, Papke K. Diagnostics and treatment of spontaneous intracranial hypotension. Minim Invasive Neurosurg 2010;53:15-20

32. Gökçay F, Eyigör C, Bayram E, et al. Epidural blood patch treatment in a patient with chronic headache related to spontaneous intracranial hypotension [in Turkish]. Agri 2010;22:170-74

33. Mokri B. Spontaneous cerebrospinal fluid leaks: from intracranial hypotension to cerebrospinal fluid hypovolemia-evolution of a concept. Mayo Clin Proc 1999;74:1113-23

34. Yildiz H, Erdogan C, Yalcin R, et al. Evaluation of communication between intracranial arachnoid cysts and cisterns with phase-contrast cine MR imaging. AJNR Am J Neuroradiol 2005;26:145-51

35. Hakyemez B, Yildiz H, Parlak M. CSF connection of the intracranial cysts and cyst like lesions: analysis with flow sensitive phase-contrast cine MR imaging [in Turkish]. Tani Girisim Radyol 2003;9:152-66

36. Algin O, Hakyemez B, Gokalp G, et al. Phase-contrast cine MRI versus MR cisternography on the evaluation of the communication between intraventricular arachnoid cysts and neighbouring cerebrospinal fluid spaces. Neuroradiology 2009;51:305-12. Epub 2009 Jan 27

37. Rekate HR. The definition and classification of hydrocephalus: personal recommendation to stimulate debate. Cerebrospinal Fluid Res 2008;5:2

38. Stoquart-El Sankari S, Lehmann P, Gondry-Jouet C, et al. Phasecontrast MR imaging support for the diagnosis of aqueductal stenosis. AJNR Am J Neuroradiol 2009;30:209-14. Epub 2008 Oct 2

39. Dinçer A, Yener U, Ozek MM. Hydrocephalus in patients with neurofibromatosis type 1: MR imaging findings and the outcome of 
endoscopic third ventriculostomy. AJNR Am J Neuroradiol 2011; 32:643-46

40. Dinçer A, Kohan S, Ozek MM. Is all "communicating" hydrocephalus really communicating? Prospective study on the value of $3 \mathrm{D}-$ constructive interference in steady state sequence at $3 \mathrm{~T}$. AJNR Am J Neuroradiol 2009;30:1898-906

41. Bailey A, Pipitone N, Zuccoli G. Phase-contrast cine MRI revealing en valve mechanism in spontaneous third ventriculostomy: report of a case and literature review. Clin Neurol Neurosurg 2010;112:81720. Epub 2010 Jul 8

42. Stachura K, Moskała M. Spontaneous third ventriculostomy in obstructive hydrocephalus with composed aetiology: a case report [in Polish]. Neurol Neurochir Pol 2010;44:87-90

43. Dinçer A, Yildiz E, Kohan S, et al. Analysis of endoscopic third ventriculostomy patency by MRI: value of different pulse sequences, the sequence parameters, and the imaging planes for investigation of flow void. Childs Nerv Syst 2011;27:127-35

44. Bilginer B, Oguz KK, Akalan N. Endoscopic third ventriculostomy for malfunction in previously shunted infants. Childs Nerv Syst 2009;25:683-88
45. Algin O. Role of complex hydrocephalus in unsuccessful endoscopic third ventriculostomy. Childs Nerv Syst 2010;26:3-4

46. Algin O, Hakyemez B, Parlak M. The efficiency of PC-MRI in diagnosis of normal pressure hydrocephalus and prediction of shunt response. Acad Radiol 2010;17:181-87

47. Algin O, Hakyemez B, Parlak M. Proton MR spectroscopy and white matter hyperintensities in idiopathic normal pressure hydrocephalus and other dementias. Br J Radiol 2010;83: $747-52$

48. Algin O, Hakyemez B, Ocakoglu G, et al. MR cisternography: Is it useful in the diagnosis of normal pressure hydrocephalus and the selection of "good shunt responders"? Diagn Interv Radiol 2011; 17:105-11

49. Algin O, Hakyemez B, Taskapilioglu O, et al. Morphologic features and flow void phenomenon in normal pressure hydrocephalus and other dementias: are they really significant? Acad Radiol 2009;16: $1373-80$

50. Algin O. Role of aqueductal CSF stroke volume in idiopathic normal-pressure hydrocephalus. AJNR Am J Neuroradiol 2010;31:E26-27 